\title{
Precision Irrigation for Sunagoke Moss Production using Intelligent Image Analysis
}

\author{
Yusuf Hendrawan and Haruhiko Murase \\ Graduate School of Life and Environmental Science, Osaka Prefecture University, \\ 1-1 Sakai, Osaka, 599-8531
}

(Received February 19, 2009)

\begin{abstract}
There are many methods for sensing water condition in Sunagoke moss Rachomitrium canescens. The direct measurement of canopy is considered to be relatively inefficient and destructive to the plant. One alternative is the use of indirect measurement and non-destructive techniques such as machine vision. This study investigated the use of machine vision for monitoring water content in Sunagoke moss. The goal of this paper was to propose and investigate a combined genetic-neural algorithm to find the most significant image features or the sets of image features suitable for predicting Sunagoke moss water content. We extracted 50 features consisting of color, textural (Gray Level Co-occurrence Matrix and RGB Color Co-occurrence Matrix textural features) and morphological features. Ten textural features were calculated, including Entropy, Energy, Contrast, Homogeneity, Sum Mean, Variance, Correlation, Maximum Probability, Inverse Difference Moment and Cluster Tendency. The specificity of this problem was that we were not looking for single feature but several associations of features that may be involved in determining water content. The genetic algorithm was able to select features with 27 selected features and artificial neural network was able to predict water content according to the selected features with minimum error of MSE 0.0021 .
\end{abstract}

Keywords : artificial neural network (ANN), feature selection (FS), genetic algorithms (GAs), machine vision, water content

\section{INTRODUCTION}

Moss water content

Sunagoke Moss Rachomitrium canescens has been utilized as an active greening material to mitigate the urban heat island (UHI) effect (Murase and Ushada, 2006; Hendrawan and Murase, 2008a). Various effects can be expected from greening wall and rooftop greening to solve mitigation of UHI. These effects include storm water storage, delay and control of rain runoff, creation and restoration of ecosystems and horticultural therapy (Park and Murase, 2007).

One of the primary determinants of Sunagoke moss growth is water availability. Different parts of Sunagoke moss have different capacities to hold and transport water. Water affects evapotranspiration (ET) and canopy photosynthesis, and hence net primary productivity (NPP). Environmental factors greatly affect ET rates on a water body. Different part of moss have different capacities to hold and transport water (Kellner, 2001).

There are many methods for sensing water condition in Sunagoke moss. The direct measure-

Corresponding author: Haruhiko Murase, fax : +81-72-254-9918, e-mail : hmurase@bics.envi.osakafu-u.ac.jp 
ment of canopy parameters is considered to be relatively inefficient, destructive to the plants (Ushada et al., 2007) and can not always provide accurate results at the large scale production of Sunagoke moss in plant factory. Even a slight contact of foreign material with the plant tissue disturbs physiological activity of the plant (Murase et al., 1997). For example, plant moisture conditions in terms of plant water potential level can be measured by extracting a small tissue from a leaf, which should be placed in a chamber of a psychrometric transducer. This method is destructive and it can affect to the physiological activity. One of alternatives is the use of indirect measurement techniques. It may be possible to recognize changes in some kind of indices that describe the water conditions from the appearance of wilting Sunagoke moss by machine vision. Shape and texture features have been used for some time for pattern recognition in datasets such as remote sensed imagery, medical imagery, photographs, etc (Newsam and Kamath, 2005). An analysis of the morphology of the plant features could be particularly useful to determine indicators that characterize the plant quality (Kurata and Yan, 1996; Recce et al., 1996; Foucher et al., 2004). Many studies have reported use combination of color, morphology and textural features to detect stress in plant (Ahmad and Reid, 1996; Escos et al., 2000; Leemans et al., 2002).

\section{Relevant feature selection}

It is possible to extract many features from Sunagoke moss image including color features, textural features and morphological features, but it is difficult to determine which feature or features subsets are relevant to measure water content of Sunagoke moss. Selecting features is critical to improving the accuracy and speed of prediction system.

Feature selection (FS) techniques can be used to overcome these difficulties. FS techniques have become an apparent need in many bioinformatics applications (Handels et al., 1999; Utku, 2000; Zheng, 2006; Zhu et al., 2007; Unay and Gosselin, 2007; Saeys et al., 2007; Verma and Zhang, 2007; Gomez-Sanchis et al., 2008; Zapotoczny et al., 2008). FS is a process that chooses an optimal subset of features according to an objective function. It is used to reduce dimensionality and remove irrelevant features and to improve mining performance. There are two aspects for FS, first is how to decide whether a feature is relevant to the objective function or not and second is how to decide whether such a relevant feature is redundant or not compared to other features.

\section{Goal and objectives}

The main goal was utilizing machine vision as non-invasive method and the combination of artificial neural network (ANN) and feature selection (FS) techniques to determine water content of Sunagoke moss. It can be subdivided into some objectives as following:

- to investigate the effect of water content to evapotranspiration process and photosynthesis process in Sunagoke moss;

- to evaluate the variability in the color, morphology and textural features of Sunagoke moss due to the change in water content;

- to select image features which are relevant for predicting Sunagoke moss water content;

\section{MATERIALS AND METHODS}

\section{Materials and equipments}

Materials

1. Cultured Sunagoke moss mat Rhacomitrium canescens of dimensions $500 \mathrm{~mm} \times 500 \mathrm{~mm}$ as shown in Fig. 1(a). The samples are made of living cultured Sunagoke moss growing in polyvinyl (PVC) netting and anchored in glass wool media. A sample of cultured Sunagoke moss (Fig. 1(b)), placed in a $110 \mathrm{~mm} \times 80 \mathrm{~mm} \times 25 \mathrm{~mm}$ glass vessel was used in this study.

2. Distilled water, in which water was given in the amount of $1 \mathrm{gg}^{-1}$ (1 gram of water content per gram of dry weight of Sunagoke moss), $2 \mathrm{gg}^{-1}, 3 \mathrm{gg}^{-1}$ and $4 \mathrm{gg}^{-1}$. Water content 


\section{INTELLIGENT IMAGE ANALYSIS}

(WC) was determined as:

$$
W C_{a v g}=\frac{t w-d w}{d w}
$$

where: $W C_{\text {avg }}$ is the average water content, $t w$ is the total weight (wet weight) and $d w$ is initial dry weight of Sunagoke moss. Dry weight of moss was determined by drying process until the weight of moss was stabilized without any further decrement.

\section{Equipments}

Moss images were taken through the digital camera (Nikon Coolpix SQ 3.1-megapixel effective CCD for images up to $2016 \times 1512$ pixels, Japan) placed at $330 \mathrm{~mm}$ perpendicular to the sample surface. The shooting parameters of the digital camera were image quality: normal and image size: $1024 \times 768$ pixels. The camera axis was vertical so that the images were the projections of the canopy. Imaging took place in a dark room under controlled and well distributed light conditions. Images, obtained in the red, green, blue (RGB) color system. Each color plane was quantified on 256 levels. A total 124 images were acquired. Light was provided by two $22 \mathrm{~W}$ lamps (EFD25N/22, National Corporation, Japan). Light intensity over the moss surface was at $100 \mu \mathrm{mol}$ $\mathrm{m}^{-2} \mathrm{~s}^{-1}$ PPF during image acquisition. Data acquisition was conducted in a walk-in growth chamber (Biotron NK 350 Big Size/Walk-In Chamber, Japan). After acquisition, ImageJ (Rasband, NH) software was used for processing the images and changed the resolution to $565 \times 400$ pixels.

Growth chamber was used to control environment parameters (air temperature ca. $15^{\circ} \mathrm{C}, \mathrm{RH}$ ca. $65 \%$, the $\mathrm{CO}_{2}$ gas ca. $400 \mu \mathrm{mol} \mathrm{mol}{ }^{-1}$, light ca. $86.5 \mu \mathrm{mol} \mathrm{m}^{-2} \mathrm{~s}^{-1}$ for $12 \mathrm{~h}$ ). We soaked the moss canopy area of $8,800 \mathrm{~mm}^{2}$ with distilled water, put them on plastic plates and left them to dry under growth chamber conditions for some several periods of time.

Photosynthesis and ET rate were measured using Li6400 (Li-Cor Biosciences Portable Photosynthesis System, USA) equipped with a $100 \mathrm{~mm}$ (height) $\times 180 \mathrm{~mm}$ (diameter) leaf tube chamber. $\mathrm{CO}_{2}$ mixer was used and it was set to control reference $\mathrm{CO}_{2}$ with a target slightly above ambient. Reference cell $\mathrm{CO}_{2}$ was controlled at $400 \mu \mathrm{mol} \mathrm{CO}_{2} \mathrm{~mol}^{-1}$. Flow rate to the sample cell was $500 \mu \mathrm{mol} \mathrm{s}^{-1}$. Leaf temperature was maintained at $25^{\circ} \mathrm{C}$ and light was maintained at $100 \mu \mathrm{mol}$ $\mathrm{m}^{-2} \mathrm{~s}^{-1} \mathrm{PPF}$ throughout the period of study. The $\mathrm{CO}_{2}$ flux was measured inside a transparent chamber. The chamber was connected to a Li6400 $\mathrm{CO}_{2}$ Analyzer (LI-COR) in a closed circuit. $\mathrm{CO}_{2}$ flux was calculated from the linear change in $\mathrm{CO}_{2}$ concentration during the first minute of measurement. In this study we observed cultured Sunagoke moss. Leaf area is simply the canopy area of cultured Sunagoke moss exposed inside the chamber $\left(8,800 \mathrm{~mm}^{2}\right)$. Stomata ratio is an estimate of the ratio of stomata on one side of the leaf to the other. Stomata ratio was set at 0 for stomata on only one side. The recording started almost immediately after deploying the chamber and the concentration was averaged over intervals of $5 \mathrm{~s}$. $\mathrm{CO}_{2}$ exchange as a function of water content was studied with total of 5 Sunagoke moss samples. The fifth samples were maintained at water content level of 0 $\mathrm{gg}^{-1}, 1 \mathrm{gg}^{-1}, 2 \mathrm{gg}^{-1}, 3 \mathrm{gg}^{-1}$ and $4 \mathrm{gg}^{-1}$. The Li6400 system measurements and formulae were used for determination of assimilation $\left(A, \mu \mathrm{mol} \mathrm{CO}_{2} \mathrm{~m}^{-2} \mathrm{~s}^{-1}\right)$ and evapotranspiration $\left(E T\right.$, mmol $\mathrm{H}_{2} \mathrm{O}$ $\left.\mathrm{m}^{-2} \mathrm{~s}^{-1}\right)$. Experiments were taken in 10 iterations.

\section{Software}

Intelligent image analysis software included features extraction and FS using wrapper method (combined genetic algorithms (GAs) and ANN) was built in Visual Basic (self-built software).

\section{Model study}

As shown in Fig. 2, the steps involved in the experimental design were:

1. Image acquisition; in which an image of Sunagoke moss under test was taken.

2. Image analysis and features extraction; extracting three features (color, morphology and texture). Color features included RGB color index and RGB mean value. Morphological features included green canopy index, perimeter index and browning area index. 
Textural features were extracted using RGB Color Co-occurrence Matrix (CCM) and Grey Level Co-occurrence Matrix (GLCM).

3. FS; in which relevant features were selected was using filter methods and wrapper method (combined genetic-neural algorithm).

4. Back-propagation Neural Network (BPNN); training the inputs for predicting water content.

5. Measurements; observing the effect of water content to optimum photosynthesis.

6. Irrigation control design; modeling a concept of intelligent image analysis application to control water content in Sunagoke moss based on optimum photosynthesis.

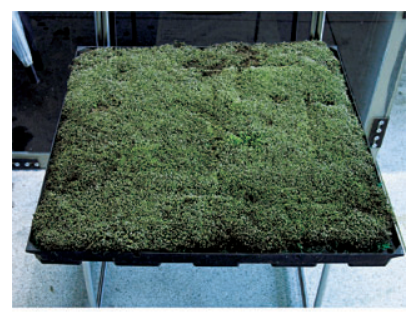

(a)

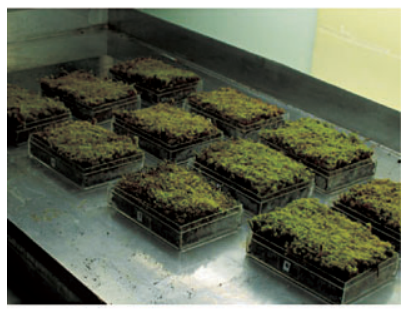

(b)

Fig. 1 Cultured Sunagoke: (a) moss mat $(500 \mathrm{~mm} \times 500 \mathrm{~mm})($ b) sample used in this study.

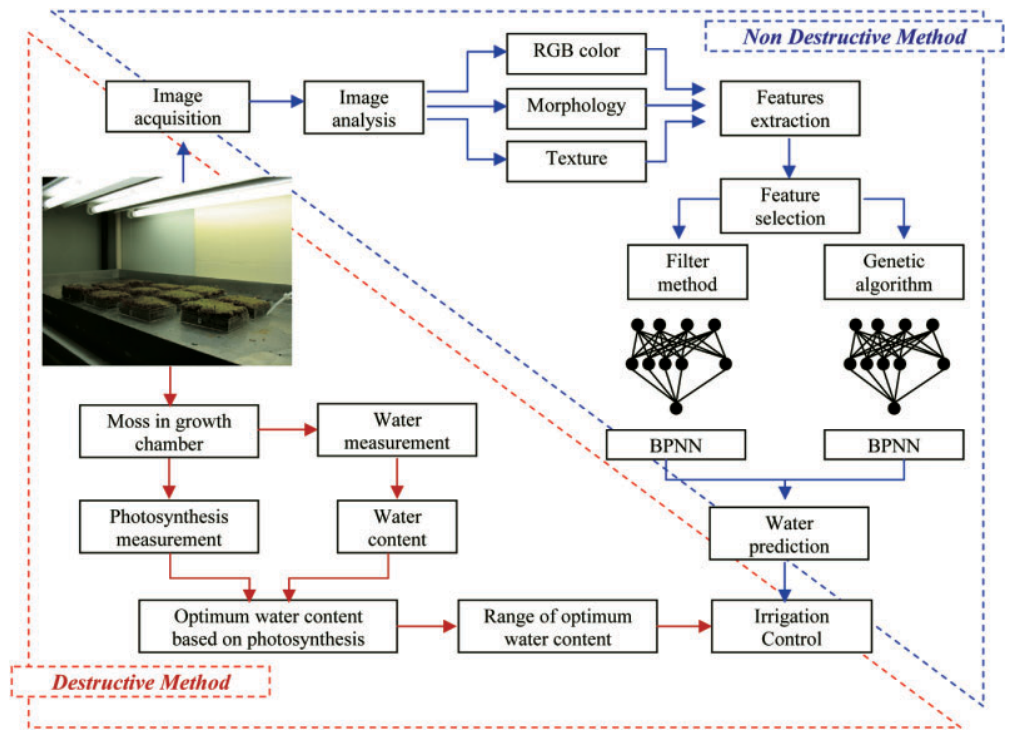

Fig. 2 Experimental design for precision irrigation system based on optimum photosynthesis. 


\section{METHODS}

\section{Features extraction}

In our system, we extract 7 color features, 3 morphological features and 40 textural features from the Sunagoke moss pictorial data. The red (R), green $(\mathrm{G})$ and blue (B) color primaries were extracted for color features. RGB CCM and GLCM were used to extract textural features. Morphological features from Sunagoke moss images including green canopy index, perimeter index and browning area index were also extracted.

In this study the features which were observed included: (1) green/red ratio variance, (2) average red index, (3) average green index, (4) average blue index, (5) red mean value, (6) green mean value, (7) blue mean value, (8) perimeter index, (9) green canopy index, (10) browning area index, (11) red energy, (12) red entropy, (13) red contrast, (14) red homogeneity, (15) red inverse difference moment, (16) red correlation, (17) red sum mean, (18) red variance, (19) red cluster tendency, (20) red maximum probability, (21) green energy, (22) green entropy, (23) green contrast, (24) green homogeneity, (25) green inverse difference moment, (26) green correlation, (27) green sum mean, (28) green variance, (29) green cluster tendency, (30) green maximum probability, (31) blue energy, (32) blue entropy, (33) blue contrast, (34) blue homogeneity, (35) blue inverse difference moment, (36) blue correlation, (37) blue sum mean, (38) blue variance, (39) blue cluster ten-
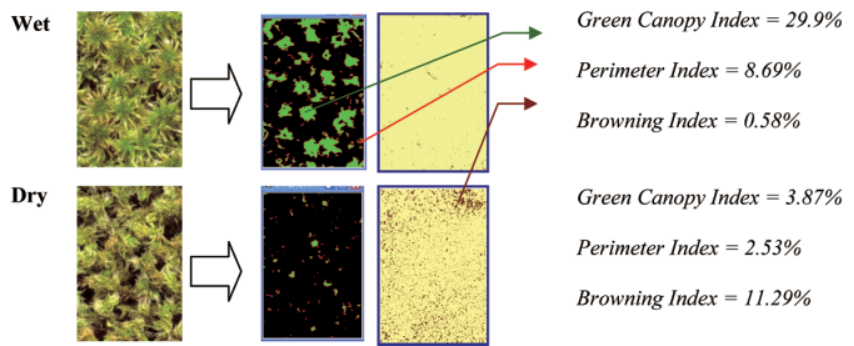

Fig. 3 Green canopy, perimeter index and browning area index in different water condition of Sunagoke moss.

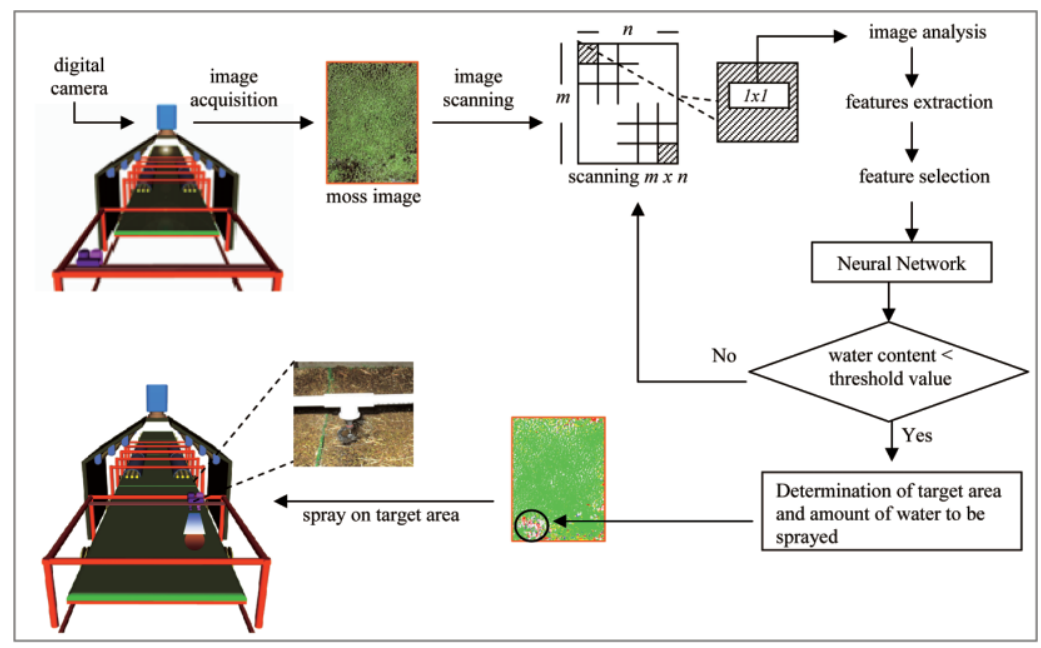

Fig. 12 Proposed irrigation control design for Sunagoke moss. 
dency, (40) blue maximum probability, (41) grey energy, (42) grey entropy, (43) grey contrast, (44) grey homogeneity, (45) grey inverse difference moment, (46) grey correlation, (47) grey sum mean, (48) grey variance, (49) grey cluster tendency, (50) grey maximum probability.

$R G B$ color components

The RGB components i.e. red index, green index, blue index, red mean value, green mean value, blue mean value and green/red ratio variance were analyzed and can be described as:

$$
\begin{aligned}
\text { Average Red Index } & =\frac{1}{N} \sum_{i=1}^{N} \frac{R}{R+G+B} \\
\text { Average Green Index } & =\frac{1}{N} \sum_{i=1}^{N} \frac{G}{R+G+B} \\
\text { Average Blue Index } & =\frac{1}{N} \sum_{i=1}^{N} \frac{B}{R+G+B} \\
\text { Red Mean Value } & =\frac{1}{N} \sum_{i=1}^{N} R \\
\text { Green Mean Value } & =\frac{1}{N} \sum_{i=1}^{N} G \\
\text { Blue Mean Value } & =\frac{1}{N} \sum_{i=1}^{N} B \\
\text { Green / Red Ratio Variance } & =\frac{1}{N} \sum_{i=1}^{N}\left(x_{i}-\bar{x}\right)^{2}
\end{aligned}
$$

where: $\mathrm{R}$ (red value); $\mathrm{G}$ (green value); B (blue value); $\mathrm{N}$ is the total number of pixels; $x_{i}$ is green/ red ratio value and $\bar{x}$ is green/red ratio mean value.

\section{Morphological features}

Morphological features extracted included browning area index, green canopy index and perimeter index. Browning process on Sunagoke moss can be influenced by virus, fungi, water content or environment condition. The threshold point of browning area index and green canopy index can be determined using green/red ratio mean value. The threshold point of browning area index is between 0.93 and 1.13 of green/red ratio mean value. The threshold point of green canopy index is above 1.71 of green/red ratio mean value (Hendrawan and Murase, 2008b). Perimeter is used in calculating the border of an object (green canopy area). Figure 3 shows that water content effects green canopy index, perimeter index and browning area index. In dry condition, green canopy and perimeter index of Sunagoke moss has lower value than Sunagoke moss which has enough water. This was reversed for browning area index.

\section{Textural features}

Texture is a measure of the variation of the intensity of a surface, quantifying properties such as smoothness, coarseness and regularity. Two-dimensional co-occurrence (gray-level dependence) matrices are generally used in texture analysis because they are able to capture the spatial dependence of gray-level values within an image (Haralick et al., 1973). The textural analysis can be considered as one of applicable techniques for extracting image features (Haralick et al., 1973; Murase et al., 1994). A $2 \mathrm{D}$ co-occurrence matrix, $P$, is an $n \times n$ matrix, where $n$ is the number of

Table 1 Sensitivity analysis of textural features for water content prediction using neural network.

\begin{tabular}{lcc}
\hline Textural Features: & \multicolumn{2}{c}{ Average Prediction Error (MSE) } \\
\cline { 2 - 3 } Red CCM, Green CCM, Blue CCM and GLCM & Training Set & Validation Set \\
\hline distance $(\mathrm{d}=1)$ and angle $(\theta=0)$ & 0.22 & 0.28 \\
distance $(\mathrm{d}=1)$ and angle $(\theta=45)$ & 0.28 & 0.37 \\
distance $(\mathrm{d}=1)$ and angle $(\theta=90)$ & 0.29 & 0.38 \\
distance $(\mathrm{d}=1)$ and angle $(\theta=135)$ & 0.26 & 0.37 \\
\hline
\end{tabular}


gray-levels within an image. The matrix acts as an accumulator so that $P[i, j]$ counts the number of pixel pairs having the intensities $i$ and $j$. Pixel pairs are defined by a distance and direction which can be represented by a displacement vector $d=(d x, d y)$, where $d x$ represents the number of pixels moved along the x-axis, and $d y$ represents the number of pixels moved along the y-axis of an image slice.

Ten textural features were calculated i.e. entropy, energy (angular second moment), contrast, homogeneity, sum mean (mean), variance, correlation, maximum probability, inverse difference moment and cluster tendency. In this study RGB CCM and GLCM textural features were used. Table 1 shows sensitivity analysis of textural features for predicting water content using BPNN (number of training-set $=112$, validation-set $=12$, iterations $=400$, nodes $=20$, learning coefficient $=0.5$ and momentum=0.5). The result shown in Table 1, average prediction MSE (training-set and validation-set) of Red CCM, Green CCM, Blue CCM and GLCM textural features shows that texture analysis using angle $(\theta=0)$ performed better than other angle. Therefore, in this study, textural features were extracted at distance $(\mathrm{d}=1)$ and angle $(\theta=0)$. The range of RGB CCM and GLCM within a given image determines the dimensions of a two-dimensional co-occurrence matrix. In each of RGB CCM and GLCM have 256 grey-levels, which would make the co-occurrence matrix $256 \times$ 256. Ten textural features used in this research can be described as following (Kurani et al., 2004):

$$
\begin{gathered}
\text { Entropy }=-\sum_{i}^{M} \sum_{j}^{N} P[i, j] \log P[i, j] \\
\text { Energy }=\sum_{i}^{M} \sum_{j}^{N} P^{2}[i, j] \\
\text { Contrast }=\sum_{i}^{M} \sum_{j}^{N}(i-j)^{2} P[i, j] \\
\text { Homogeneity }=\sum_{i}^{M} \sum_{j}^{N} \frac{P[i, j]}{1+[i-j]} \\
\text { Sun Mean }=\frac{1}{2} \sum_{i}^{M} \sum_{j}^{N}(i P[i, j]+j P[i, j]) \\
\text { Variance }=\frac{1}{2} \sum_{i}^{M} \sum_{j}^{N}\left((i-\mu)^{2} P[i, j]+(j-\mu)^{2} P[i, j]\right) \\
\text { Correlation }=\sum_{i}^{M} \sum_{j}^{N} \frac{(i-\mu)(j-\mu) P[i, j]}{\sigma^{2}} \\
\text { Maximum } \operatorname{Pr} \text { obability }=\operatorname{Max}_{i, j}^{M, N} P[i, j] \\
\text { Inverse Difference Moment }=\sum_{i}^{M} \sum_{j}^{N} \frac{P[i, j]}{|i-j|^{k}} \quad i \neq j \\
\text { Cluster Tendency }=\sum_{i}^{M} \sum_{j}^{N}(i+j-2 \mu)^{k} P[i, j]
\end{gathered}
$$

where: $P(i, j)$ is the $(i, j)^{t h}$ element of a normalized co-occurrence matrix, and $\mu$ and $\sigma$ are the mean and standard deviation of the pixel element given by the following g relationships:

$$
\begin{gathered}
P[i, j]=\frac{N(i, j)}{M} \\
\mu=\sum_{i}^{M} i \sum_{j}^{N} P[i, j] \\
\sigma=\sum_{i}^{M}(i-\mu)^{2} \sum_{j}^{N} P[i, j]
\end{gathered}
$$

where: $N(i, j)$ is the number counts in the image with pixel intensity $i$ followed by pixel intensity $j$ at one pixel displacement to the left, and $M$ is the total number of pixels. 


\section{Feature selection}

Selecting features that are suitable for an application is one of the most important parts in solving the problem. Shape descriptors (morphological features), color features and texture measures are all able to represent some information about image. The main problem is first to find efficient features for image representation, then to find effective measure that use these representation, individually or as a combination.

As many pattern recognition techniques were originally not designed to cope with large amounts of irrelevant features, combining them with FS techniques has become a necessity in many applications. The objectives of FS are manifold, the most important ones being: a) to avoid overfitting and improve model performance, i.e. prediction performance in the case of supervised classification and better cluster detection in the case of clustering, b) to provide faster and more costeffective models, and c) to gain a deeper insight into the underlying processes that generated the data (Saeys et al., 2007).

FS techniques can be organized into three categories depending on how they combine the FS search with the construction of the classification model: filter methods, wrapper methods and embedded methods. In this study we focus to solve FS problem using filter and wrapper methods. Filter techniques assess the relevance of features by looking only at the intrinsic properties of the data. Advantages of filter techniques are that they easily scale to very high-dimensional datasets, they are computationally simple and fast, and they are independent of the classification algorithm. A common disadvantage of filter methods is that they ignore the interaction with the classifier. Whereas filter techniques treat the problem of finding a good feature subset independently of the model selection step, wrapper methods embed the model hypothesis search within the feature subset search. Advantages of wrapper approaches include the interaction between feature subset search and model selection, and the ability to take into account feature dependencies. A common disadvantage of these techniques is that they have a higher risk of over fitting than filter techniques and are very computationally intensive.

In this study, we evaluate the performance of five FS methods corresponding to these approach in order to select the most contribute features to determine water content of Sunagoke moss. The five FS methods were: Chi-Squared $\left(X^{2}\right)$, Mutual Information (MI), Correlation-based Feature Selection (CFS), Linear Regression (LR) in which can be included as filter techniques and combined genetic-neural algorithm which can be included as wrapper technique.

The Chi-Squared $\left(X^{2}\right)$ method evaluates features individually by measuring their chi-squared statistic with respect to the classes (Liu et al., 2002). For numeric attribute, the method first requires its range to be discretized into several intervals using, for example, the entropy-based discretization method. After calculating the $X^{2}$ value of all considered features, we can sort these values with the largest one at the first position, as the larger the $X^{2}$ value, the more important the feature is. The $X^{2}$ value of an attribute is defined as:

$$
\begin{gathered}
X^{2}=\sum_{i=1}^{m} \sum_{j=1}^{k} \frac{\left(A_{i, j}-E_{i, j}\right)^{2}}{E_{i, j}} \\
E_{i, j}=\frac{R_{i} C_{j}}{N}
\end{gathered}
$$

where: $m$ is the number of intervals, $k$ the number of classes, $A_{i j}$ the number of samples in the $i$ th interval, $j$ th class, $R i$ the number of samples in the $i$ th interval, $C j$ the number of samples in the $j$ th class, $N$ the total number of samples, and $E_{i j}$ the expected frequency of $A_{i j}$.

MI function is suitable for assessing the information content of features (Gomez-Sanchis et al., 2008). We evaluated the mutual information (I) function for each feature and class variable, and the features with the highest mutual information function value were selected. The mutual information of two random variables $x$ and $y$ can be viewed as a quantity measuring the mutual 


\section{INTELLIGENT IMAGE ANALYSIS}

dependence of the two variables (Shannon, 1948). The mutual information is widely used in applications area of FS for machine learning. The mutual information between two random variables $x$ and $y$ can be computed as indicated:

$$
I(x ; y)=\sum_{x, y} P(x, y) \log \frac{P(x, y)}{P(x) P(y)}
$$

where: $P(x, y)$ is the joint probability distribution function of $x$ and $y$, and $P(x)$ and $P(y)$ are the marginal probability distribution functions of $x$ and $y$ respectively.

The CFS method is another approach to FS. Rather than scoring (and ranking) individual features, the method scores (and ranks) the worth of subsets of features. As the feature subset space is usually huge, CFS uses a best-first-search heuristic. This heuristic algorithm takes into account the usefulness of individual features for predicting the class along with the level of intercorrelation among them. CFS first calculates a matrix of feature-class and feature-feature correlations from the training data. Then a score of a subset of features assigned by the heuristic is defined as:

$$
\text { Merit }_{s}=\frac{\overline{k r_{c f}}}{\sqrt{k+k(k-1) \overline{r_{f f}}}}
$$

where: Merit $t_{s}$ is the heuristic merit of a feature subset $S$ containing $k$ features. $\overline{r_{c f}}$ is the average feature-class correlation, and $\overline{r_{f f}}$ is the average feature-feature intercorrelation.

Another method is using linear regression. LR analyzes the relationship between two variables, $\mathrm{X}$ and $\mathrm{Y}$. In general, the goal of LR is to find the line that best predicts Y from X. LR does this by finding the line that minimizes the sum of the squares of the vertical distances of the points from the line. The LR is defined as:

$$
Y=a+b x
$$

where: the coefficients $a$ and $b$ are determined by the condition that the sum of the square residuals is as small as possible.

The results of $X^{2}$, MI, CFS and LR were feature ranking which ranked the features based on the relevancy to the predicted variable (water content). BPNN was used to train selected features based on the ranking number to find relevant combination of features for predicting water content. BPNN configuration for filter methods and genetic-neural algorithm method included number of training-set $=112$, validation-set $=12$, iterations $=400$, nodes $=20$, learning coefficient $=0.5$ and

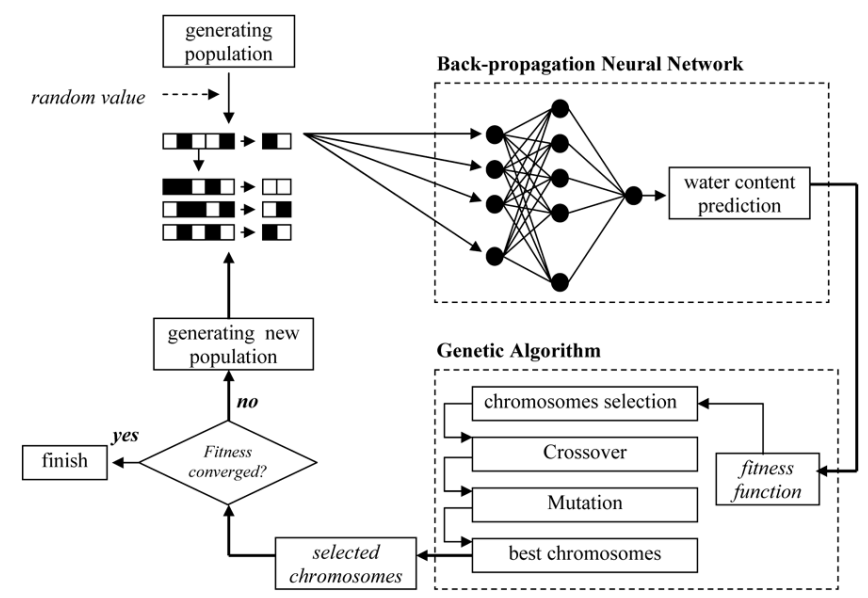

Fig. 4 Feature selection using combined genetic-neural algorithm. 
momentum $=0.5$. The total samples used in this study were 124 samples and for the observation, we soaked the moss samples with distilled water of $0^{-} 0.5 \mathrm{gg}^{-1}$ (16 samples as training-set and 1 as validation-set), $0.5^{-1} \mathrm{gg}^{-1}$ (16 samples as training-set and 2 as validation-set), $1^{-1.5} \mathrm{gg}^{-1}$ (16 samples as training-set and 2 as validation-set), $1.5^{-2} \mathrm{gg}^{-1}$ (16 samples as training-set and 2 as validation-set), 2-2.5 $\mathrm{gg}^{-1}$ (16 samples as training-set and 2 as validation-set), $2.5^{-3} \mathrm{gg}^{-1}$ (16 samples as training-set and 2 as validation-set), 3-3.5 gg (16 samples as training-set and 1 as validation-set).

GAs are search algorithms based on the mechanics of natural selection and natural genetics (Goldberg, 1997). GAs are different from more normal optimization and search procedures in four ways: (1) GAs work with a coding of the parameter set, not the parameters themselves; (2) GAs search from a population of points, not a single point; (3) GAs use payoff (objective function) information, not derivatives or other auxiliary knowledge; and (4) GAs use probabilistic transition rules, not deterministic rules.

In this research, a combined genetic-neural algorithm was developed for FS based on the neural network pattern recognition as shown in Fig. 4. Each individual in the population represents a candidate solution to the feature subset selection problem. There were $\left(2^{50}-1\right)$ possible feature subsets. The values of the BPNN inputs were the normalized image features that are between 0 and 1. One output of BPNN was used to determine Sunagoke moss water content. The selected features are the inputs of the BPNN, which are used for water content prediction. In GAs for FS involves many generations. In each generation, evaluation of an individual (a feature subset) involves training neural networks. In this step, a binary vector of dimension 50 represents the individual in the population. The chromosome define contains 50 genes, one gene for each feature, which can take 2 values. A value of 0 indicates that the corresponding feature is not selected, while a value 1 means that the feature is selected. An initial population of chromosomes is randomly generated. Crossover was performed by 2 points real value crossover. Two points were selected randomly. Mutation process was also conducted randomly. Some best chromosomes were kept to be used in the next generation. Looping process was done until the fitness function converged to find best feature subset. The roulette wheel selection strategy was also used in the algorithm for FS. The parameter settings were: population size: 50 ; probability of best chromosomes: 0.2 ; probability of crossover: 0.8 ; probability of mutation: 0.01 and number of generation was developed until the fitness function converged. The fitness of the chromosome was calculated according to the prediction rate of the selected subset of features for predicting Sunagoke moss water content.

\section{RESULTS AND DISCUSSION}

\section{Evapotranspiration and photosynthesis}

The photosynthesis and ET of Sunagoke moss at different water contents and $\mathrm{CO}_{2}$ concentrations was measured in the laboratory. ET (Fig. 5) is much higher when the moss layer is wet. The

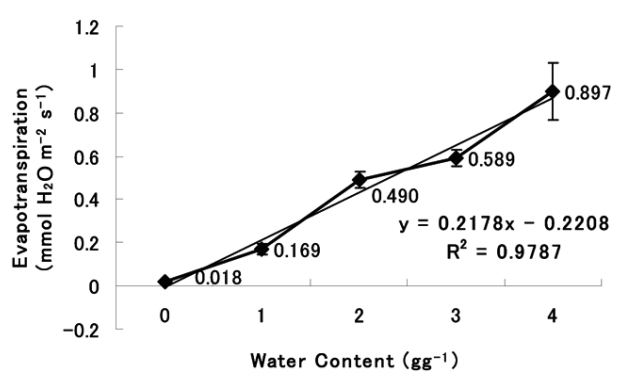

Fig. 5 Evapotranspiration process and water content. 


\section{INTELLIGENT IMAGE ANALYSIS}

ET process is composed of evaporation and transpiration. Water content has a linier correlation with ET. It means that as water content increases, the ET rate also increases.

One major question concerning the direct effects of optimum water content is the increase in photosynthesis and production rates, or the possibility of some limitation of this production increase due to other environmental factors. Optimum condition of Sunagoke moss water content as

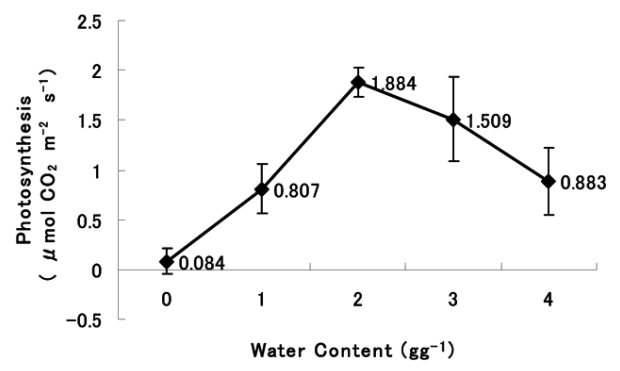

Fig. 6 Photosynthesis process and water content.

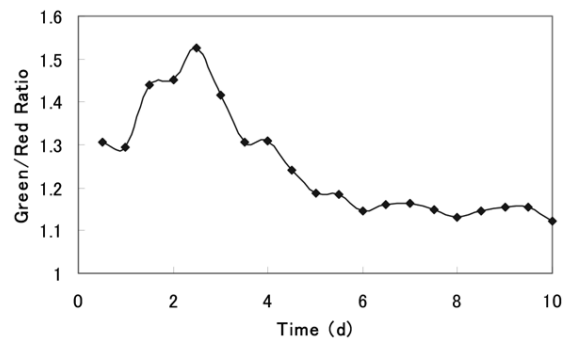

(a)

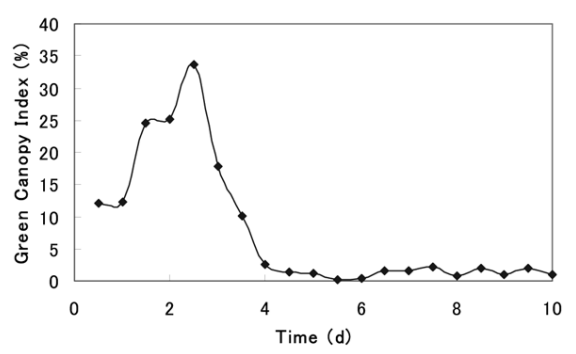

(b)

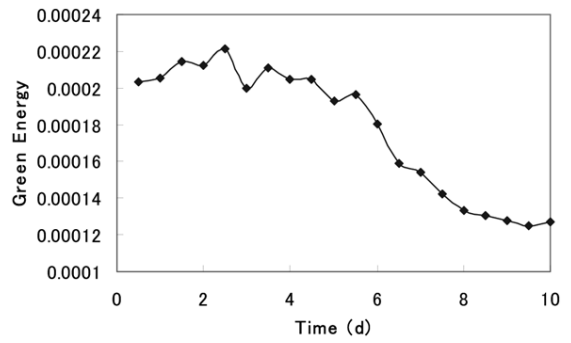

(c)

Fig. 7 Drying process based on (a) green/red ratio, (b) green canopy index, (c) green energy.

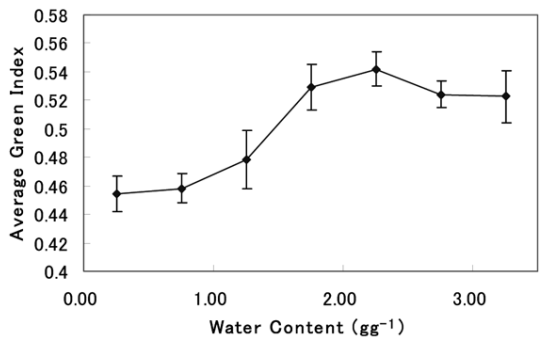

(a)

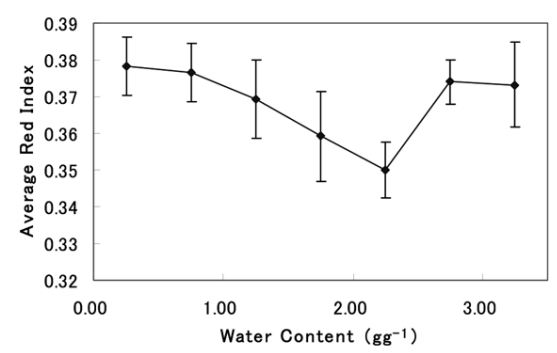

(b)

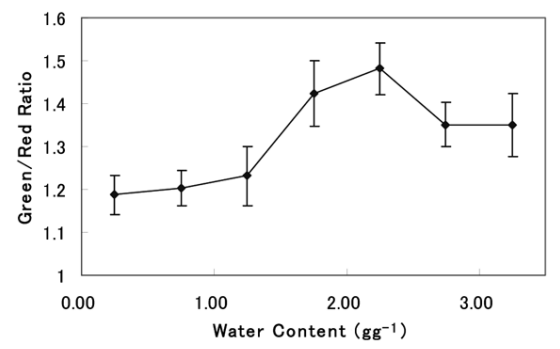

(c)

Fig. 8 RGB color features: (a) average green index, (b) average red index and (c) green/red ratio. 
shown in Fig. 6 based on photosynthesis rate (light in the environment growth condition: $100 \mu \mathrm{mol}$ $\mathrm{m}^{-2} \mathrm{~s}^{-1}$ ) was achieved at $2 \mathrm{gg}^{-1}$. The decrease in photosynthesis is very steep towards lower water contents and less steep towards higher water contents. In moss saturated with water, photosynthesis increases gradually until $1.884 \mu \mathrm{mol} \mathrm{CO} \mathrm{Cm}^{-2} \mathrm{~s}^{-1}$ when the water content was $2 \mathrm{gg}^{-1}$, but photosynthesis becomes lower with increasing water content, being $0.883 \mu \mathrm{mol} \mathrm{CO}_{2} \mathrm{~m}^{-2} \mathrm{~s}^{-1}$.

Color, morphology and textural features

Figure 7 shows the pattern comparison of color (a), morphology (b) and textural (c) features during drying process in 10 days experiment. Color feature (green/red ratio mean value) shows the trend of decreasing value with the peak point in day 2.5 which the water content reached approximately $2 \mathrm{gg}^{-1}$. Morphology (green canopy index) and textural feature (green energy) also have the same condition with color feature.

\section{Color features and water content}

Visible light photography has been effective in determining the percentage of maximal net $\mathrm{CO}_{2}$ uptake using green/red ratio (Graham et al., 2006). Red referred to a broad band of wavelength $\left(600^{-699} \mathrm{~nm}\right)$ and green to a broad band of wavelength $\left(500^{-} 599 \mathrm{~nm}\right)$. During the photosynthesis process, plant absorbs red wavelength that makes it reflect red wavelength less than green wavelength, so the more it absorb red wavelength the higher green/red ratio value, while photosyn-

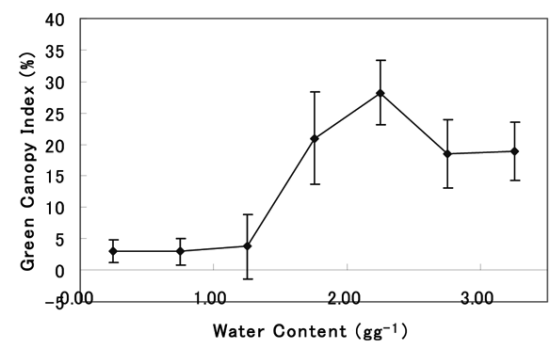

(a)

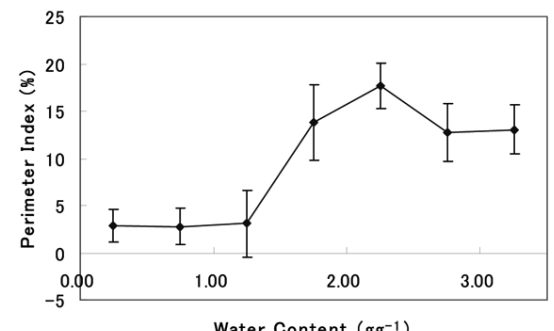

(b)

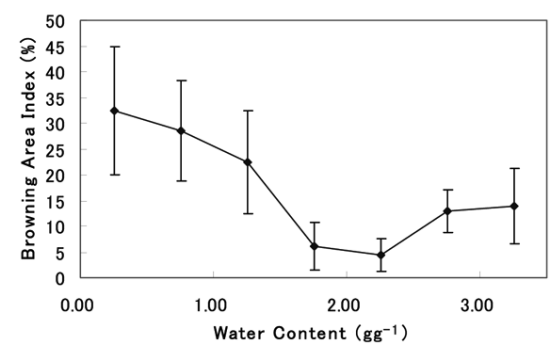

(c)

Fig. 9 Morphological features: (a) green canopy index, (b) perimeter index, (c) browning area index. 


\section{INTELLIGENT IMAGE ANALYSIS}

thesis has relation with appropriate water existence. If Sunagoke moss does not have enough water or it has too much water, then photosynthesis process will not be optimum. Photosynthesis of Sunagoke moss will be optimum if it has appropriate water content as shown in Fig. 6. The graph of different levels of water content (Fig. 8) shows that generally Sunagoke moss has peak point of color features (average green index, average red index and green/red ratio mean value) between 2 $\mathrm{gg}^{-1}$ and $2.5 \mathrm{gg}^{-1}$ water. Average blue index and blue mean value had linier correlation with water content.

\section{Morphological features and water content}

Green canopy index in Fig. 9 (a) and perimeter index in Fig. 9 (b) reached the optimum point between $2 \mathrm{gg}^{-1}$ and $2.5 \mathrm{gg}^{-1}$ of water content. Figure 9 (c) shows that water content influences the browning process of Sunagoke moss. Water condition affects to browning process. Condition in which moss does not get enough water or it contains too much water, the browning process increase and raises the browning area index value. In dry condition, browning area index was high, but then it decreased gradually until $4.4 \%$ in water condition between $2 \mathrm{gg}^{-1}$ and $2.5 \mathrm{gg}^{-1}$, but it became higher again with increasing water content, being $12.9 \%$ and went fairly stable after the water condition had reached $2.75 \mathrm{gg}^{-1}$.

\section{Textural features and water content}

Table 2 shows the average of green CCM textural features of images for the Sunagoke moss samples. All green CCM textural features except sum mean textural features had a peak point at the water content between $2 \mathrm{gg}^{-1}$ and $2.5 \mathrm{gg}^{-1}$. From Table 2, energy, homogeneity, inverse difference moment and maximum probability textural features increased up to the water content between $2 \mathrm{gg}^{-1}$ and $2.5 \mathrm{gg}^{-1}$, remained fairly stable after water condition had reached $2.5 \mathrm{gg}^{-1}$. This was reversed for entropy, contrast, correlation, variance and cluster tendency. Hence optimum condition of Sunagoke moss based on color, morphology and textural features can be achieved between $2 \mathrm{gg}^{-1}$ and $2.5 \mathrm{gg}^{-1}$ water content.

\section{Feature selection using combined genetic-neural algorithm}

The research was conducted by the prediction rate based on validation set to calculate the fit-

Table 2 Green CCM textural features and Sunagoke moss water content.

\begin{tabular}{|c|c|c|c|c|c|c|c|c|c|c|}
\hline \multirow[b]{2}{*}{ Water } & \multicolumn{10}{|c|}{ Average Green CCM Textural Features } \\
\hline & Energy & Entropy & Contrast & $\begin{array}{c}\text { Homoge- } \\
\text { neity }\end{array}$ & Inverse & Correlation & Sum Mean & Variance & $\begin{array}{l}\text { Cluster } \\
\text { Tendency }\end{array}$ & $\begin{array}{c}\text { Max } \\
\text { Probability }\end{array}$ \\
\hline $0-0.5$ & $1.3 \times 10^{-4}$ & 4.03 & $4.78 \times 10^{-2}$ & $1.21 \times 10^{-1}$ & $6.68 \times 10^{-2}$ & $4.38 \times 10^{-7}$ & $1.17 \times 10^{-2}$ & $1.07 \times 10^{-3}$ & $3.79 \times 10^{-3}$ & $3.91 \times 10^{-4}$ \\
\hline $0.5^{-1}$ & $1.35 \times 10^{-4}$ & 4.01 & $4.66 \times 10^{-2}$ & $1.21 \times 10^{-1}$ & $6.69 \times 10^{-2}$ & $3.86 \times 10^{-7}$ & $1.19 \times 10^{-2}$ & $9.89 \times 10^{-2}$ & $3.49 \times 10^{-3}$ & $3.52 \times 10^{-4}$ \\
\hline $1^{-1.5}$ & $1.78 \times 10^{-4}$ & 3.90 & $3.93 \times 10^{-2}$ & $1.28 \times 10^{-1}$ & $7.22 \times 10^{-2}$ & $2.01 \times 10^{-7}$ & $1.25 \times 10^{-2}$ & $7.23 \times 10^{-2}$ & $2.50 \times 10^{-3}$ & $4.51 \times 10^{-4}$ \\
\hline $1.5^{-2}$ & $2.00 \times 10^{-4}$ & 3.86 & $3.90 \times 10^{-2}$ & $1.29 \times 10^{-1}$ & $7.38 \times 10^{-2}$ & $1.41 \times 10^{-7}$ & $1.26 \times 10^{-2}$ & $6.21 \times 10^{-2}$ & $2.09 \times 10^{-3}$ & $5.23 \times 10^{-4}$ \\
\hline $2-2.5$ & $2.07 \times 10^{-4}$ & 3.85 & $3.74 \times 10^{-2}$ & $1.32 \times 10^{-1}$ & $7.55 \times 10^{-2}$ & $1.38 \times 10^{-7}$ & $1.21 \times 10^{-2}$ & $6.09 \times 10^{-2}$ & $2.06 \times 10^{-3}$ & $5.29 \times 10^{-4}$ \\
\hline $2.5^{-3}$ & $1.95 \times 10^{-4}$ & 3.87 & $3.93 \times 10^{-2}$ & $1.30 \times 10^{-1}$ & $7.41 \times 10^{-2}$ & $1.49 \times 10^{-7}$ & $1.05 \times 10^{-2}$ & $6.35 \times 10^{-2}$ & $2.15 \times 10^{-3}$ & $5.14 \times 10^{-4}$ \\
\hline $3-3.5$ & $1.95 \times 10^{-4}$ & 3.87 & $4.03 \times 10^{-2}$ & $1.29 \times 10^{-1}$ & $7.38 \times 10^{-2}$ & $1.40 \times 10^{-7}$ & $1.02 \times 10^{-2}$ & $6.17 \times 10^{-2}$ & $2.06 \times 10^{-3}$ & $5.05 \times 10^{-4}$ \\
\hline
\end{tabular}

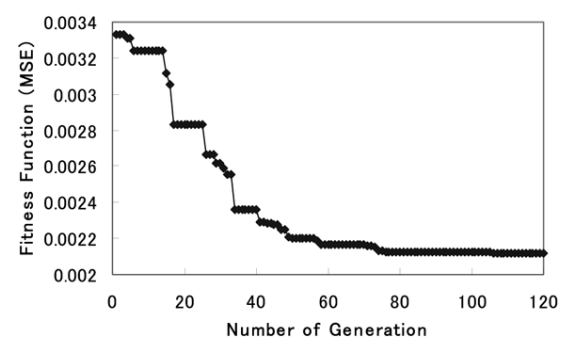

Fig. 10 Fitness functions of genetic algorithm for feature selection. 


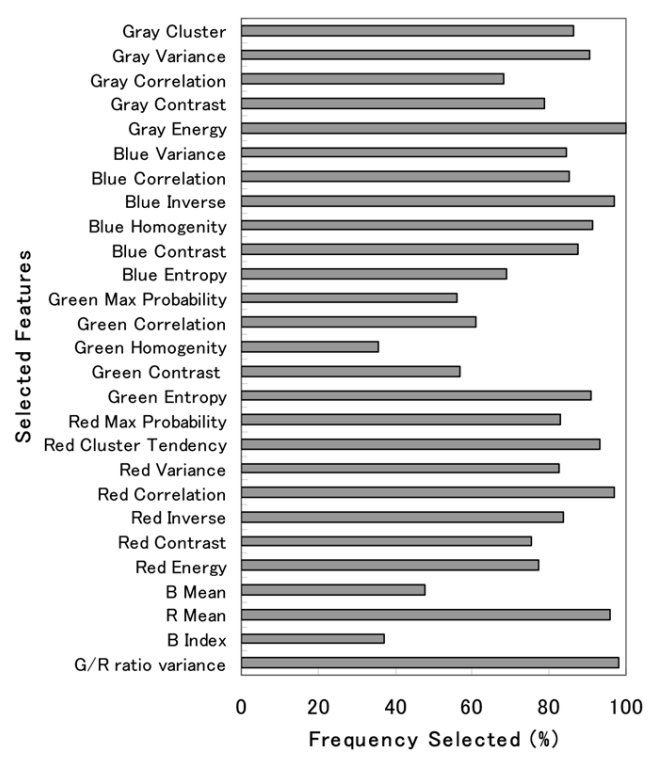

Fig. 11 The selection rate of every selected feature.

Table 3 Comparison analysis of five feature selection methods.

\begin{tabular}{lccc}
\hline \multirow{2}{*}{ Feature Selection Method } & \multirow{2}{*}{$\begin{array}{c}\text { Number of } \\
\text { Selected Features }\end{array}$} & \multicolumn{2}{c}{ Prediction Error (MSE) } \\
\cline { 3 - 4 } & 50 & $3.1 \times 10^{-2}$ & $4.0 \times 10^{-2}$ \\
\hline All features with no Feature Selection & 36 & $2.8 \times 10^{-2}$ & $2.9 \times 10^{-2}$ \\
Chi-Squared $\left(X^{2}\right)$ & 31 & $2.9 \times 10^{-2}$ & $3.6 \times 10^{-2}$ \\
Mutual Information $(M I)$ & 17 & $3.5 \times 10^{-2}$ & $3.6 \times 10^{-2}$ \\
Correlation based Feature Selection (CFS) & 22 & $2.4 \times 10^{-2}$ & $3.3 \times 10^{-2}$ \\
Linear Regression $(L R)$ & 27 & $5.4 \times 10^{-3}$ & $2.1 \times 10^{-3}$ \\
Genetic-Neural Algorithm & & & \\
\hline
\end{tabular}

ness for reproduction of GAs. The number of hidden layer (1 hidden layer) and hidden nodes (20 nodes) were determined to be effectively used in BPNN structures to achieve the best prediction of water content. The results of FS are described as following. First of all, in most cases, the accuracy of prediction performance using five proposed FS method were greatly improved. It was shown in Table 3 that there were some improvements in case of number of features used and the prediction error between methods using FS and method without FS.

Among the filter methods, $X^{2}$ method got the highest performance for prediction with the validation error $2.9 \times 10^{-2}$ and 36 features selected followed by LR with validation error $3.3 \times 10^{-2}$ and 22 features selected. Overall the results, the experiments which were conducted using filter method, the results were much worse than using wrapper method (genetic-neural algorithm). When analyzing the results of the genetic-neural algorithm, it is noticed that the lowest prediction validation error (MSE: $2.1 \times 10^{-3}$ ) was achieved by BPNN with a few sets of selected features ( 27 features). Figure 10 shows the fitness function of GAs, it converged after 120 generations using 50 populations in each generation. Figure 11 shows the frequency selected for each relevant feature in every generation. There were nine most selected features with the frequency selected rate above $90 \%$. The most contributed features to predict water content were: grey energy with frequency selected $100 \%$, green/red ratio variance $(98.2 \%)$, blue inverse difference moment $(97.1 \%)$, red correlation $(96.9 \%)$, red mean value $(95.7 \%)$, red cluster tendency $(93 \%)$, blue homogeneity $(91.2 \%)$, green entropy $(91.1 \%)$ and grey variance $(90.6 \%)$. Genetic-neural algorithm has successfully 


\section{INTELLIGENT IMAGE ANALYSIS}

selected relevant image features to determine water content of Sunagoke moss.

Neural network based precision irrigation for sunagoke moss

The proposed irrigation control design of Sunagoke moss is shown in Fig. 12. Pictorial information from Sunagoke moss (27 relevant features) can be utilized as the input of BPNN to detect dry area and the output is the amount lack of water. The water sprayer will irrigate the dry part of Sunagoke moss in accurate places and accurate amount of water. The decrement of dry area and optimum water condition can bring Sunagoke moss into optimum photosynthesis process. The main objective of this precision irrigation system is to optimize the water supply of Sunagoke moss and stabilize the amount of water content between $2 \mathrm{gg}^{-1}$ and $2.5 \mathrm{gg}^{-1}$.

\section{CONCLUSIONS}

In conclusions, optimum condition of Sunagoke moss water content based on photosynthesis rate (light in the environment growth condition: $100 \mu \mathrm{mol} \mathrm{m} \mathrm{m}^{-2} \mathrm{~s}^{-1}$ ) was achieved at $2 \mathrm{gg}^{-1}$. Optimum condition of Sunagoke moss water content based on color features, morphological features and textural features were achieved between $2 \mathrm{gg}^{-1}$ and $2.5 \mathrm{gg}^{-1}$ water content. FS is very important for predicting Sunagoke moss water content. We have proposed and implemented a novel approach for finding the most significant set of image features to predict water content of Sunagoke moss. The achieved minimum prediction error and feature sets are promising. The minimum prediction error achieved for validation set was $2.1 \times 10^{-3}$ with 27 features selected. For most experiments, BPNN architecture of one hidden layer with 20 nodes is most suitable for prediction. Genetic-neural algorithm for FS techniques is effective compare to others ( $X^{2}$, MI, CFS and LR). Though, Sunagoke moss was used in this study, this method can be extended to other plants. Finally, neural network based precision irrigation system has been proposed to optimize the water supply of Sunagoke moss and stabilize the amount of water content between $2 \mathrm{gg}^{-1}$ and $2.5 \mathrm{gg}^{-1}$. This system is applicable for Sunagoke moss production based on controlled environment such as plant factory.

\section{REFERENCES}

Ahmad, I.S., Reid J. F. 1996. Evaluation of color representations for maize images. J. Agr. Eng. Res. 63: 185-196.

Escos, J., Alados C. L., Pugnaire F.I., Pigdefabregas J., Emlen, J. 2000. Stress resistance strategy in an arid land shrub: interactions between developmental instability and fractal dimension. J. Arid Environ. 45: 325336.

Foucher, P., Revollon, P., Vigouroux, B., Chasseriaux, G. 2004. Morphological image analysis for the detection of water stress in potted forsythia. Biosyst. Eng. 89: 131-138.

Goldberg, D. E. 1997. Genetic algorithm in search, optimization and machine learning. Addison Wesley Longman, Inc, USA, pp 1-15.

Gomez-Sanchis, J., Gomez-Chova, L., Aleixos, N., Camps-Valls, G., Montesinos-Herrero, C., Molto, E., Blasco, J. 2008. Hyperspectral system for early detection of rottenness caused by Penicillium digitatum in mandarins. J. Food Eng. 89: 80-86.

Graham, E. A., Hamilton, M. P., Mishler, B. D., Rundel, P. W., Hansen, M. H. 2006. Use of a network digital camera to estimate net $\mathrm{CO}_{2}$ uptake of a desiccation-tolerant moss. Int. J. Plant Sci. 167: 751-758.

Handels, H., Rob, T., Kreusch, J., Wolff, H. H., Poppl, S. j. $1999 . \quad$ Feature selection for optimized skin tumor recognition using genetic algorithms. Artif. Intell. Med. 16: 283-297.

Haralick, R. M., Shanmugam, K., Dinstein, Its'hak. 1973. Textural features for image classification. IEEE T. Syst. Man Cyb. 3: 610-621.

Hendrawan, Y., Murase, H. 2008a. Intelligent irrigation control using color, morphological and textural features in Sunagoke Moss. An ASABE Annual International Meeting, Providence, USA, June 29-July 2, 
paper number: 083858 .

Hendrawan, Y., Murase, H. 2008b. Water irrigation control for Sunagoke Moss using intelligent image analysis. 17th IFAC World Congress Proceedings, Seoul, Korea, July 6-11, paper number: 2723.

Kellner, E. 2001. Surface energy fluxes and control of evapotranspiration from Swedish sphagnum mire. Agr. Forest Meteorol. 110: 101-123.

Kurani, A. S., Xu, D. H., Furst, J. D., Raicu, D. S. 2004. Co-occurrence matrices for volumetric data. The 7th IASTED International Conference on Computer Graphics and Imaging - CGIM, Kauai, Hawaii, USA, in August 16-18.

Kurata, K., Yan, J. 1996. Water stress estimation of tomato canopy based on machine vision. Acta Hort. 440: 389-394.

Leemans, V., Magein, H., Destain, M. F. 2002. On-line fruit grading according to their external quality using machine vision. Biosyst. Eng. 83: 397-404.

Liu, H., Li, J., Wong, L. 2002. A comparative study on feature selection and classification methods using gene expression profiles and protemic patterns. Genome Informatics 13: 51-60.

Murase, H., Honami, N., Nishiura, Y. 1994. Image information feedback using textural features for plant growth control. Proceedings of the First Asian Control Conference, Tokyo, 27-30 July, Vol. 3, pp 17-20.

Murase, H., Ushada, M. 2006. Machine vision applications for micro-precision agriculture. Environ. Control Biol. 44: 199-206.

Murase, H., Nishiura, Y., Mitani, K. 1997. Environmental control strategies based on plant responses using intelligent machine vision technique. Comput. Electron. Agric. 18: 137-148.

Newsam, S., Kamath, C. 2005. Comparing shape and texture features for pattern recognition in simulation data. IS\&T/SPIE's Annual Symposium on Electronic Imaging San Jose, CA, United States, January 16-20.

Park, J. E., Murase, H. 2007. Forced convection airflow driven by massive evaporation of water from moss served as a cover plant for building surfaces. An ASABE Annual International Meeting, Minneapolis, USA, June 17-20, paper number: 077129.

Recce, M., Taylor, J., Plebe, A., Tropiano, G. 1996. High speed vision-based quality grading of oranges. International Workshop on Neural Networks for Identification, Control, Robotics and Signal/Image Processing, IEEE Computer Society Press, Venice, Italy, pp 136-144.

Saeys, Y., Inza, I., Larranaga, P. 2007. A review of feature selection techniques in bioinformatics. Bioinformatics 23: 2507-2517.

Shannon, C. E. 1948. A mathematical theory of communication. AT\&T. Tech. J. 27, pp 379-423, 623-656.

Unay, D., Gosselin, B. 2007. Stem and calyx recognition on 'Jonagold' apples by pattern recognition. J. Food Eng. 78: 597-605.

Ushada, M., Murase, H., Fukuda, H. 2007. Non-destructive sensing and its inverse model for canopy parameters using texture analysis and artificial neural network. Comput. Electron. Agric. 57: 149-165.

Utku, H. 2000. Application of the feature selection method to discriminate digitized wheat varieties. J. Food Eng. 46: 211-216.

Verma, B., Zhang, P. 2007. A novel neural-genetic algorithm to find the most significant combination of features in digital mammograms. Appl. Soft Comput. 7: 612-625.

Zapotoczny, P., Zielinska, M., Nita, Z. 2008. Application of image analysis for the varietal classification of barley: morphological features. J. Cereal Sci. 48: 104-110.

Zheng, C., Sun, D. W., Zheng, L. 2006. Correlating color to moisture content of large cooked beef joints by computer vision. J.Food Eng. 77: 858-863.

Zhu, Z., Ong, Y. S., Dash, M. 2007. Markov blanket-embedded genetic algorithm for gene selection. Pattern Recogn. 40: 3236-3248. 